\title{
SURFACE X-RAY ABSORPTION SPECTROSCOPY: BASIC CONCEPTS AND APPLICATIONS
}

\author{
P. LAGaRde
}

LURE Bat. 209d, Centre Universitaire Paris-Sud, 91405 Orsay, France

The application to surface problems of X-ray spectroscopy has started almost at the same time as in materials science. While the theoretical basis is obviously the same, SEXAFS has some experimental peculiarities which are reviewed here. Some examples will then be given.

PACS numbers: 78.70.Dm, 68.35.Gy

\section{Introduction}

Surface EXAFS is born almost at the same time as usual, bulk oriented EXAFS technique itself. Actually, one of the first papers involving the possibility of adsorbate site determination by monitoring the photoabsorption cross-section by means of Auger electrons is written by Lee [1] only a few months after the basic principles of EXAFS were published. It is a theoretical investigation; the experimental proof will come one year later, with the study of the adsorption site of iodine on the $\mathrm{Ag}(111)$ surface [2,3]. An extensive review ending in 1986 can be found in the paper by Citrin [4], but since that time this field has had a noteworthy growth and it would be an enormous task to do the same job today.

\section{Theoretical basis of the technique}

In terms of theory itself, surface EXAFS, so-called SEXAFS since 1977 by Citrin, does not depart from its three-dimensional counterpart. Both are described by the usual formula issued from the Fermi golden rule taking into account the modulations of the photoelectron final state due to interference effects between the outgoing wave function and the scattered parts coming from neighbouring atoms. Let us recall here this formula: the oscillating part $X(E)$ superimposed to the smooth background absorption is given by

$$
X(k)=-\sum_{i} \frac{1}{k R_{i}^{2}} N_{i}^{*} \mathrm{e}^{-2 R_{i} / \lambda} \mathrm{e}^{-2 \sigma_{i}^{2} k^{2}}\left|f_{i}(\pi)\right| \sin \left(2 k R_{i}+2 \delta_{1}+\Phi_{i}\right) .
$$

The sum over the index $i$ means a sum over different shells of neighbouring atoms, i.e. atoms of different $Z$ values or atoms at different interatomic distances $R_{i}$. These atoms are characterized by their scattering properties through 
the backscattering function $f(\pi)$ which has a modulus $|f(\pi)|$ and a phase $\phi$. The fluctuations of the interatomic distances with respect to their equilibrium position $R_{i}$ are described here, within the harmonic approximation, by a Debye-Walker-like term $\mathrm{e}^{-2 \sigma_{i}^{2} k^{2}}$, and the elastic mean free path of the photoelectron is labelled $\lambda$.

In surface EXAFS studies, where model compounds are scarcely available, phase shift functions are usually extracted from theoretical calculations, and the most recent ones use the state of the art interatomic potential modelling and the spherical wave formalism [5].

For three-dimensional systems with a cubic symmetry around the absorbing atom, $N_{i}^{*}$, the apparent coordination number is equal to the true coordination number $N_{i}$ of each shell. This cubic symmetry is obviously not respected in surface studies, and we must pay here more attention to this point since the study of the angular dependence of $N_{i}^{*}$ will basically be used for a precise site determination.

1) The case of a $K$-edge absorption. From the Fermi golden rule, an absorption initiating from an $s$ shell ( $K$-edge) with angular momentum $l=0$ will end to a state with $l=1$ ( $p$-state) only. If $\theta$ is the angle between the electric vector of the photon and the interatomic distance of interest, the correct expression for the apparent coordination number $N_{i}^{*}$ is

$$
N_{i}^{*}=\sum 3 \cos ^{2} \theta,
$$

where the sum runs over all the atoms of the given shell $i$. We see that, for a spherical symmetry, since $\left\langle\cos ^{2} \theta\right\rangle=1 / 3$, we just recover the exact number of atoms in the shell $i$.

But, in the case of a surface study, this averaging is not possible and $N_{i}^{*}$ may be different when the electric vector $E$ is set differently with respect to characteristic axis of the system. Separate experiments, on the same sample wisely oriented versus the electric field will then allow a more definite determination of an adsorbate site. Examples will be given later.

2) The $L_{2}-L_{3}$ edges cases. Because the $K$-edge of the element of interest falls outside the energy domain of the experimental setup, one can be led to use the $L$-edge absorption of this element. In that case, the EXAFS formula is more complicated since the dipole selection rule allows transitions to $d$-states (with $\Delta l=1$ ) or to $s$-state (with $\Delta l=-1$ ). The full expression writes

$$
\begin{aligned}
& X(k)=\sum \frac{F(k)}{k R^{2}} \mathrm{e}^{-2 \sigma^{2} k^{2}} \mathrm{e}^{-2 R / \lambda}\left(\left|M_{21}\right|^{2}+\frac{1}{2}\left|M_{01}\right|^{2}\right)^{-1} \\
& \times\left\{\frac{1}{2}\left(1+3 \cos ^{2} \Theta\right)\left|M_{21}\right|^{2} \sin \left(2 k R+2 \delta_{2}+\Phi\right)+\frac{1}{2}\left|M_{01}\right|^{2} \sin \left(2 k R+2 \delta_{0}+\Phi\right)\right. \\
& \left.+\left|M_{01}\right|\left|M_{21}\right|\left(1-3 \cos ^{2} \Theta\right) \sin \left(2 k R+\delta_{0}+\delta_{2}+\Phi\right)\right\},
\end{aligned}
$$

where the first term within the brackets corresponds to the $p \rightarrow d$ transition, the second one to the $p \rightarrow s$, and third term mixes both transitions. In this formula, the $M_{i j}$ terms represent the transition probabilities, and the $\phi$ terms are the phase of the backscattering functions for the given value of the final orbital number. Relative magnitudes of the $M$ values are still the subject of discussion [6], 
nevertheless the second term of $X(k)$ is small, and the third one averages to zero for a cubic symmetry. Then, in cubic systems or in powders, an $L$-edge EXAFS data can be analyzed in the same way as a $K$-edge. But, here again, the situation is more complicated when this symmetry is broken, as it is in surface studies and the whole expression should then be taken into account.

Finally, one must also be aware that surface interatomic potentials are strongly anharmonic, and this has to be taken into account in the way one analyses the data.

\section{Experimental problems}

Surface physics has obviously many characteristic features when compared to material science, or solid state chemistry. The main one concerns the sample environment, that is to say the ultrahigh vacuum. This constraint is a classical one, and all surface experiments have to overcome it. From an X-ray spectroscopy point of view, more limitative is the kind of samples which one wants to study: usually, they are single crystals of metals, oxides or semiconductors, and we are interested on the few top monolayers, or on a few monolayers of adsorbate. Then, the amount of element of interest is extremely small. All this makes a simple absorption scheme inoperative, and we have to choose an alternative detection method: total electron yield (TEY), partial electron yield (PY), Auger yield (A) or fluorescence detection (FY), each one having qualities and pitfalls. Table gathers the characteristics of these different detection schemes.

TABLE

Qualities (top) and pitfalls (bottom) of the different detection methods.

\begin{tabular}{l|l|l}
\hline \hline \multicolumn{1}{c|}{ TEY } & \multicolumn{1}{|c|}{ PY, A } & \multicolumn{1}{|c}{ FY } \\
\hline - simplicity & - surface sensitivity & - signal/background \\
- low cost & & - sensitivity for high $Z$ \\
- signal/noise & & \\
- non UHV & & - diffraction peaks \\
\hline - absolute sensitivity & - cost & for high $Z$ \\
- signal/background & - interference with \\
& $\begin{array}{l}\text { photoemission peaks } \\
\text { and substrate } \\
\text { diffraction peaks }\end{array}$ & - cost for low $Z$ \\
\end{tabular}

In surface problems, an important criterion may be the depth sensitivity. FY has no surface sensitivity, since usually the mean absorption length of an $\mathrm{X}$-ray photon amounts several thousands of $\AA$. But, by the use of a solid state detector with a good energy resolution, one can discard all unwanted signal but the fluorescence of interest. On the other hand, Auger and partial yield detections have this high sensitivity when one can tune the band pass of the detector to an energy close to that of the minimum electron mean free path. Nevertheless, these two last methods may suffer of interferences with photoemission peaks entering 
the energy window, and they usually have the poorest signal to noise ratio. At the contrary, TEY is less surface sensitive, since the electron flux comes from several hundreds of $\AA$ due to inelastic processes, the collected signal is high, but the jump at the edge of interest, from the studied overlayers, is smeared out by the high background from the substrate. Nevertheless, TEY is probably the most widely used method, because of its low cost (just measure the drain current from the sample) and because it shows the higher overall signal/noise ratio. One of the first use of the different sampling depths of these data collection modes was done by Comin et al. [7] on a silicon crystal amorphized by ion bombardment. The data were collected with three different modes: Auger yield, using the KLL (about $1500 \mathrm{eV}$ ) or $L V V$ (about $90 \mathrm{eV}$ ) lines, and total electron yield. TEY data resembles that from pure crystalline silicon, since it comes from a region of the sample deep underneath the surface which has not been amorphized. $K L L$-Auger data shows the amorphous layer whose thickness corresponds approximately to the escape depth of the $K L L$ electrons, while $L V V$ Auger electrons at $90 \mathrm{eV}$ have the shortest sampling depth. More recently, we have measured [8] the relative jump at the edge of thin layers of silicon of thicknesses ranging from 5 to $500 \AA$ deposited onto $\mathrm{CaF}_{2}$ and one can conclude that most of the signal collected by TEY on crystalline silicon comes from the first $500 \AA$ below the surface. This value has to be compared with the elastic mean free path of silicon Auger $L V V$ electrons which is about $5 \AA$.

The proportionality of the signal obtained by these various schemes to the true absorption coefficient $\mu$ has been addressed experimentally and theoretically. While the self-absorption effect might be important in FY, it is usually not a problem in surface EXAFS where the sample thickness is very low, except in some cases of very high absorptions as for $M_{4-5}$ edges of rare earths. In the "thin limit", when the sampling depth of TEY is small compared to the absorption length of the photon, TEY collected over $4 \pi$ is a good measure of $\mu$. This is always the case, except here again for the $M_{4-5}$ edges of rare earths. Auger electrons is a de-excitation process competitive with fluorescence, this method usually gives a true value of $\mu$, while this assessment is less grounded in the case of PY.

As was discussed theoretically, a precise structural determination will take advantage of different experiments where the (usually) linearly polarized photon beam impinges the sample surface at different incident angles in order to switch on or off the contributions from different interatomic bonds. Therefore, the sample holder must allow various rotations of the sample in order to align different crystallographic axis with the horizontal electric field. The following examples will show a full use of these possibilities.

\section{A few examples of applications}

Most of the applications of X-ray spectroscopy to surface science deals with the determination of the adsorption site of an atom, or a molecule, on a well characterized crystal surface. In that sense, these studies appear as the first step of a much wider goal, for instance the understanding of the heterojunctions or multilayers behaviour, the understanding of the bonding process, or that of catalytic properties etc. The three following examples were chosen to enlight these points. 
Some other surface EXAFS experiments are focused to the surface structure itself, while also materials science may use experimental techniques very similar to those of SEXAFS.

\subsection{Adsorption site of silver on the $\mathrm{MgO}(100)$ face}

The very early stages of the $\mathrm{Ag} / \mathrm{MgO}(100)$ interface growth have been the subject of many studies since it appears as a testing ground of the metal-oxide interface structure. Surface EXAFS has been used [9] at the silver $L_{3}$ edge, in order to bring an experimental answer to the following questions: (i) are the silver atoms, at very low coverage, in registry with the $\mathrm{MgO}$ lattice despite the $4 \%$ mismatch between the two unit cells, (ii) what is the adsorption site, atop oxygen, atop magnesium or fourfold hollow site? Data obtained on one equivalent silver monolayer, with the photon electric field either parallel or perpendicular to the surface normal, are compared in Fig. 1 with the result of a similar experiment on bulk silver. One sees that, for the two surface data, the main peak, attributed to $\mathrm{Ag}-\mathrm{Ag}$ interatomic distances, is shifted toward higher values in order to match that of the substrate. Moreover, the peak at about $2 \AA$ (uncorrected from phase shifts) at grazing incidence can be analyzed as a contribution from substrate oxygen atoms, which then appears as the adsorption site of silver.

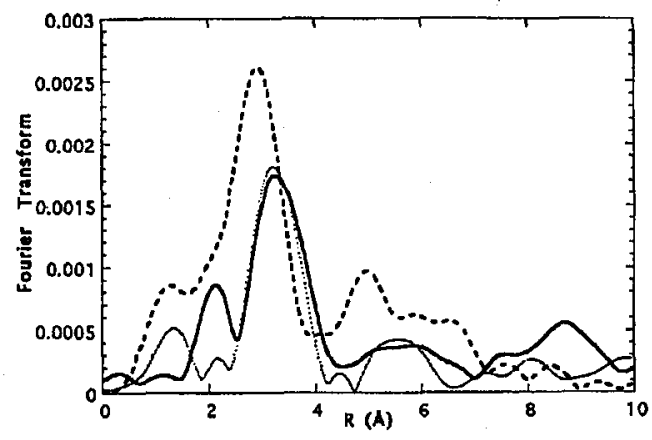

Fig. 1. Fourier transforms of the $\mathrm{Ag} L_{3}$ edge EXAFS for bulk silver (dashed line), 1 monolayer of $\mathrm{Ag} / \mathrm{MgO}(100)$ at normal incidence (solid line) and grazing incidence (dots).

\subsection{Adsorption site and growth of Co on $\mathrm{Cu}(111)$}

This study [10] belongs to the more general one of the determination of the structure of magnetic thin films and multilayers. Bulk cobalt is hcp, but the symmetry of the $\mathrm{Cu}(111)$ substrate face is that of the hcp $\mathrm{Co}(0001)$ surface, then the first problem the authors have addressed is the adsorption site of cobalt at the very early stages, using a multiple scattering analysis of the data taken on a 0.5 ML Co deposit. Because of the different symmetry around an atom deposited on an fcc continuation (ABCa sequence) compared to the ABCb one, the EXAFS signal must be different. This is illustrated in Fig. 2 where the experimental result is faced to two calculations for the two different geometries. The comparison shows unambiguously that the $\mathrm{ABCa}$ sequence is the correct one. 


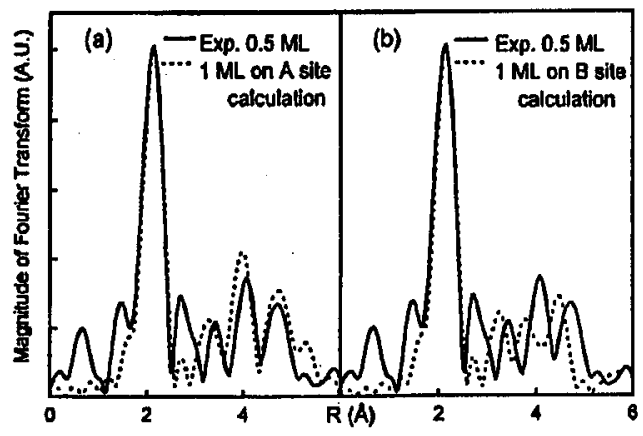

Fig. 2. Fourier transforms of the data taken at grazing incidence on $0.5 \mathrm{ML} \mathrm{Co} / \mathrm{Cu}(111)$ and of the theoretical calculations assuming the sequences $\mathrm{ABCa}$ (a) or $\mathrm{ABCb}(\mathrm{b})$.

Starting from this result, a further growth of Co might be of a fcc type (ABCabc) or a hcp one (ABCaba or ABCaca). An analysis similar to the previous one, using also the polarization of the photon beam, shows that the most probable stacking is the ABCaba one. This study is probably the first one using a multiple scattering analysis of a surface EXAFS problem, by the use of the FEFF code [11].

\subsection{Adsorption of acrylonitrile on Pt(111) and Au(111)}

This study [12], which is typical of what can be learned by X-ray spectroscopy on the interaction between a molecule and a metal surface, is not strictly speaking an EXAFS one. Rather, it deals with the near edge structure which gives

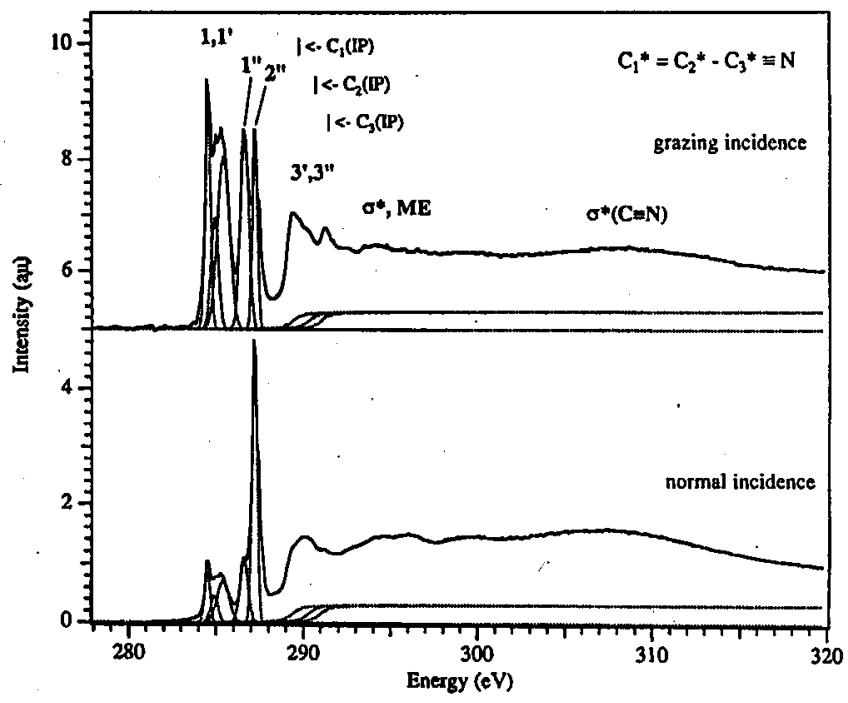

Fig. 3. X-ray absorption spectra of an acrylonitrile multilayer deposited on $\mathrm{Pt}(111)$ at the carbon $K$-edge. The upper part presents the grazing incidence spectra and the lower part is for the normal incidence. 
information about the geometry of the system, but also about the electronic interactions. X-ray absorption spectra are made at the carbon or the nitrogen $K$-edge. Both exhibit sharp resonances which are related to electronic transitions between the $1 s$ levels and unoccupied orbitals with various symmetries. A polarized study allows then, like in the EXAFS case, to determine how these molecules are placed onto the well known surface substrate since, by comparison with XPS results and $a b$ initio calculations, these transitions can be attributed to $\pi$ or $s$ orbitals. For instance, in Fig. 3, the first peak at the carbon $K$-edge $(284.5 \mathrm{eV})$ corresponds to the transition from the $\mathrm{Cl}(1 s)$ level to a $\pi_{1 z}^{*}$ orbital. Its intensity being larger for grazing incidence, it means that the molecules of the multilayer are oriented almost flat on the surface since this orbital is normal to the molecule plane.

Going now to a monolayer, X-ray absorption shows only little differences with the multilayer spectra in the $\mathrm{Au}(111)$ case, which means that the molecule is only physisorbed while, for $\mathrm{Pt}(111)$, changes are observed mostly at the nitrogen $K$-edge, a signature of a strong interaction between the molecule and the metal via this atom.

\section{Conclusion}

As bulk X-ray spectroscopy, surface EXAFS is now a well established technique which has brought much valuable information on different adsorption problems. It usually needs a very high photon flux - remember that one monolayer corresponds to about $10^{14}$ atoms $/ \mathrm{cm}^{2}$ - and surface physics has always been very expensive. Probably more than the other applications of EXAFS, this technique needs the help of complementary in situ characterization methods. All this explains why, on synchrotron radiation facilities, SEXAFS projects take much more time than the other studies.

\section{References}

[1] P.A. Lee, Phys. Rev. B 13, 5261 (1976).

[2] P.H. Citrin, Bull.Am. Phys. Soc. 22, 359 (1977).

[3] P.H. Citrin, P. Eisenberger, R.C. Hewitt, J. Vac. Sci. Technol. 15, 449 (1978).

[4] P.H. Citrin, J. Phys. C 8, 437 (1986).

[5] J.J. Rehr, S.I. Zabinsky, R.C. Albers, Phys. Rev. Lett. 69, 3397 (1992).

[6] P. Le Fèvre, H. Magnan, D. Chandesris, Phys. Rev. B 54, 2830 (1996).

[7] F. Comin, L. Incoccia, P. Lagarde, G. Rossi, P.H. Citrin, Phys. Rev. Lett. 54, 122 (1985).

[8] M.L. Hasnaoui, A.M. Flank, M. Pompa, P. Lagarde, J. Vac. Sci. Technol. 14, 2275 (1996).

[9] A.M. Flank, R. Delaunay, P. Lagarde, M. Pompa, J. Jupille, Phys. Rev. B 53, R1737 (1996).

[10] P. Le Fèvre, H. Magnan, O. Heckmann, V. Briois, D. Chandesris, Phys. Rev. B 52, 11462 (1995).

[11] J.J. Rehr, Jpn. J. Appl. Phys. Suppl. 62-8, 8 (1993).

[12] Ph. Parent, C. Laffon, G. Tourillon, A. Cassuto, J. Phys. Chem. 99, 5058 (1995). 